Cambridge University Press

978-0-521-69670-8 - Obstetrics for Anaesthetists

Edited by John Clift and Alexander Heazell

Excerpt

More information

CHAPTER ONE

Maternal physiology and obstetrics

John Clift

\title{
Overview of obstetrics
}

Obstetrics describes care related to pregnancy. In high-risk cases, such as maternal cardiac or renal disease, this may include pre-pregnancy care to optimise the mother's medical condition prior to conception. Care continues throughout the antenatal period, differing for women depending on their risk status (described in Chapter 2). Obstetric care then focuses on labour and delivery, and continues to the end of the postpartum period. During this time there are profound changes in maternal physiology. In addition, disorders may develop that are unique to pregnancy e.g. pre-eclampsia, obstetric cholestasis. An understanding of the changes in maternal physiology and the pathophysiology of pregnancy-related disorders is essential to provide safe, effective obstetric care.

\section{Maternal physiology}

There are many good textbooks describing the physiological changes occurring in pregnancy and these changes are beyond the scope of this book. This chapter summarises the implications these changes will have on anaesthetic practice.

\section{Cardiovascular and haematological system}

\section{Changes to maternal cardiac physiology}

- $\uparrow$ cardiac output (CO) (Figure 1.1), $\uparrow$ stroke volume, $\uparrow$ heart rate, $\downarrow$ systemic vascular resistance in pregnancy

- Left ventricular hypertrophy and dilatation

- Blood pressure alters throughout pregnancy (Figure 1.2)

Patients with pre-existing cardiovascular disease decompensate during pregnancy and may develop cardiac failure. Auto-transfusion due to uterine emptying at delivery causes $\uparrow$ venous return, which may precipitate cardiac failure in susceptible patients.

- Patients with cardiovascular disease need close monitoring and multidisciplinary care throughout their pregnancy with the involvement of obstetricians, anaesthetists, intensivists and cardiologists

Obstetrics for Anaesthetists, ed. Alexander Heazell and John Clift. Published by Cambridge University Press. (C) Cambridge University Press 2008 


\section{Cambridge University Press}

978-0-521-69670-8 - Obstetrics for Anaesthetists

Edited by John Clift and Alexander Heazell

Excerpt

More information

\section{Chapter 1}

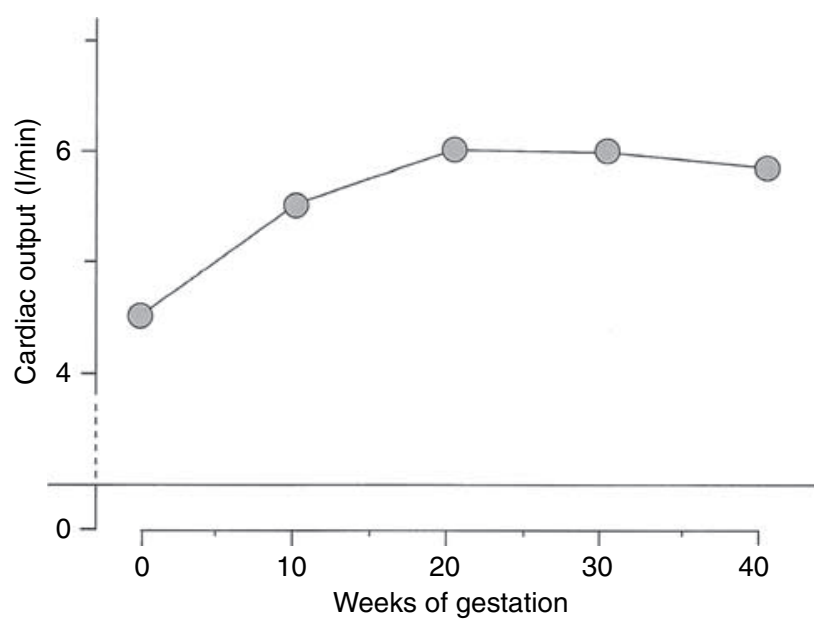

Figure 1.1 Cardiac output increases during the first trimester of pregnancy, remaining elevated throughout gestation. (Reproduced with permission from $A B C$ of Antenatal Care, 4th edn, Blackwell Publishing, 2002.) $)^{1}$

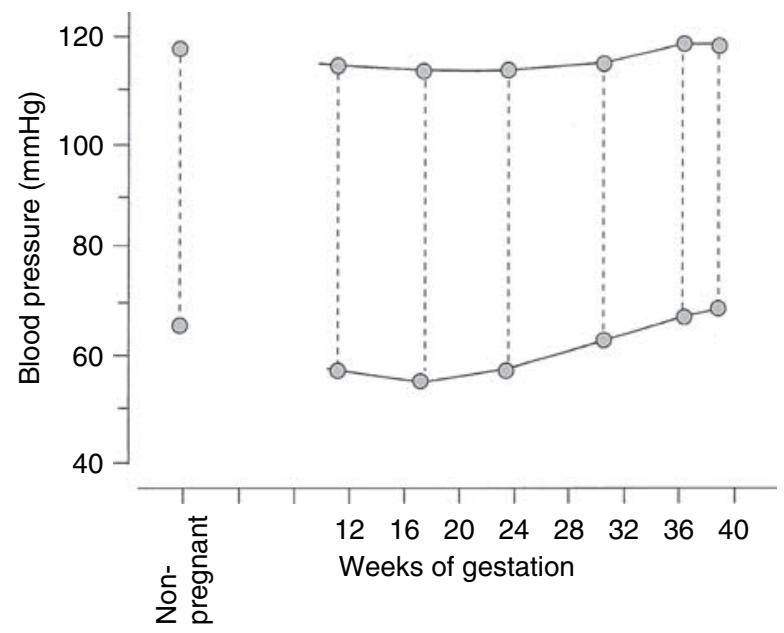

Figure 1.2 Blood pressure changes throughout pregnancy, falling during the first trimester, until approximately 20 weeks gestation, increasing towards or slightly above normal levels at 40 weeks gestation. (Reproduced with permission from $A B C$ of Antenatal Care, 4th edn, Blackwell Publishing, 2002.) ${ }^{1}$ 
Cambridge University Press

978-0-521-69670-8 - Obstetrics for Anaesthetists

Edited by John Clift and Alexander Heazell

Excerpt

More information

Maternal physiology and obstetrics

\section{Uteroplacental circulation}

- Receives $20 \%$ maternal CO at term

- Possesses no autoregulation properties

- Maternal hypotension, vasoconstriction and hypertonic uterine contractions decrease uteroplacental perfusion and may precipitate fetal hypoxia and distress

\section{ECG}

- Left axis deviation

- T waves may be inverted in lateral leads and lead III

- Mild tricuspid regurgitation may cause a grade I or II systolic murmur

\section{Supine hypotensive syndrome}

- Occurs from mid-pregnancy

- Gravid uterus compresses IVC, decreasing venous return and CO

- May cause placental hypoperfusion and maternal hypotension

- Avoid supine position, use left lateral position or left lateral tilt

- Worse with polyhydramnios and multiple pregnancies

- May be unmasked by regional or general anaesthesia

\section{Blood pressure measurement in pregnancy}

Mercury sphygmomanometry remains the most accurate method of measuring, although several automated machines have been validated to measure blood pressure in pregnancy.

- The optimal position is sitting although measuring the blood pressure in the left arm with the patient in the left lateral position is a useful alternative

- The diastolic blood pressure should be taken as the pressure at which the sounds disappear (the 5th Korotkov sound)

- A large cuff should be used if the patient's arm is greater than $33 \mathrm{~cm}$ in circumference

\section{Circulating volume}

- $45 \% \uparrow$ plasma volume, $20 \% \uparrow$ red cell mass, $\downarrow$ haemoglobin (Hb) from 13.3 to $10.9 \mathrm{~g} / \mathrm{dl}=$ physiological anaemia of pregnancy (Figure 1.3)

- $\uparrow$ plasma volume delays the onset of signs and symptoms of hypovolaemia

- $\uparrow$ circulating volume allows compensation for average blood loss at vaginal delivery $(300 \mathrm{ml})$ and Caesarean section $(500 \mathrm{ml})$, negating the need for blood transfusion

\section{Haematology}

- $\uparrow$ WCC up to $10.5 \times 10^{9} / 1$ in late pregnancy and as high as $20 \times 10^{9} / 1$ in labour

- There is a slight $\downarrow$ platelet count during pregnancy 


\section{Cambridge University Press}

978-0-521-69670-8 - Obstetrics for Anaesthetists

Edited by John Clift and Alexander Heazell

Excerpt

More information

\section{Chapter 1}

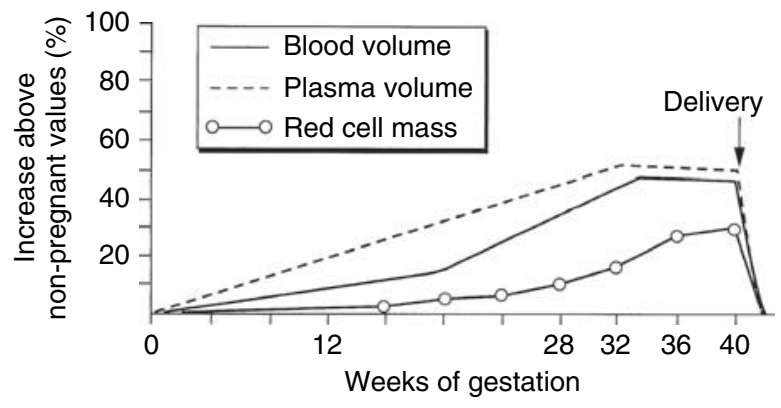

Figure 1.3 Increases in volume of constituents of blood during pregnancy, indicating that the largest component is expansion of plasma volume. (Reproduced with permission from $A B C$ of Antenatal Care, 4th edn, Blackwell Publishing, 2002.) ${ }^{1}$

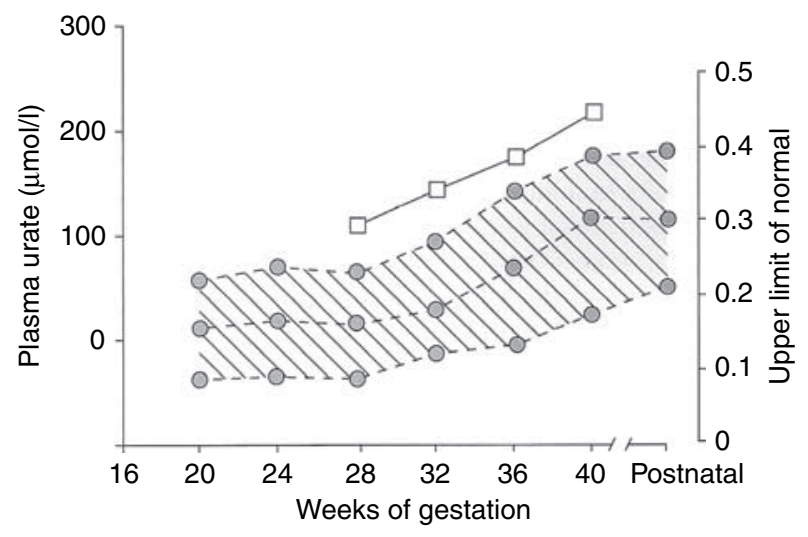

Figure 1.4 Concentration of urate in maternal plasma increases from the 20th week of gestation. There is a wide range of normal levels. O indicates the 10th, 50th and 90th centile values respectively; $\square$ indicates elevated levels present in severe pre-eclampsia. (Reproduced with permission from $A B C$ of Antenatal Care, 4th edn, Blackwell Publishing, 2002. $)^{1}$

\section{Clotting}

- Patients are hypercoagulable to facilitate clotting at the time of placental separation and prevent bleeding during pregnancy

- $\uparrow$ all factors except XI and XIII

- Thromboembolic disease is leading cause of maternal mortality in the UK 


\section{Cambridge University Press}

978-0-521-69670-8 - Obstetrics for Anaesthetists

Edited by John Clift and Alexander Heazell

Excerpt

More information

\section{Maternal physiology and obstetrics}

- Consider thromboprophylaxis and/or early mobilisation in all patients

- $\uparrow$ risk of sagittal vein thrombosis

\section{Biochemistry}

- $\downarrow$ plasma urea and creatinine levels due to $\uparrow$ renal excretion

- Plasma urate 0.15-0.35 mmol/l (gestation dependent - see Figure 1.4)

- Liver enzymes are slightly elevated with a larger $\uparrow$ alkaline phosphatase secreted from placenta

- $\downarrow \mathrm{Ca}^{2+}$

\section{Respiratory system}

\section{Oxygenation}

- Functional residual capacity (FRC) $\downarrow$ by $20 \%, \uparrow \mathrm{O}_{2}$ consumption by $20 \%$ at term (60\% in labour) (Figure 1.5)

- Women desaturate much quicker when apnoeic (during attempts at intubation or eclamptic seizure)

- A pre-oxygenation period of 3-5 minutes is recommended before general anaesthetic

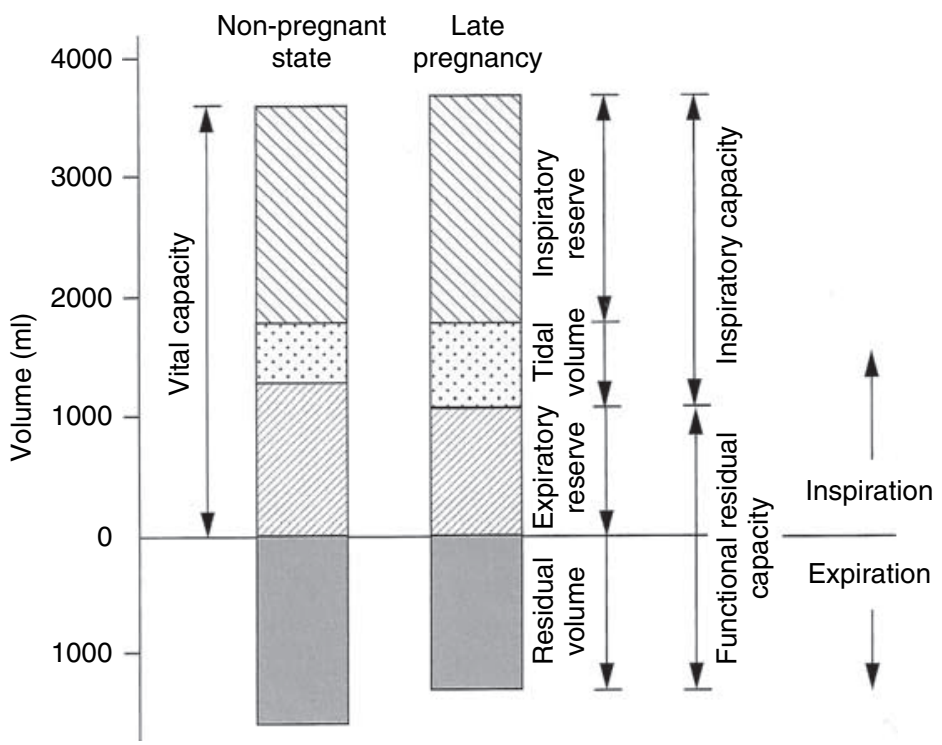

Figure 1.5 Changes in ventilatory volumes during pregnancy. (Reproduced with permission from $A B C$ of Antenatol Care, 4th edn, Blackwell Publishing, 2002.) ${ }^{1}$ 


\section{Cambridge University Press}

978-0-521-69670-8 - Obstetrics for Anaesthetists

Edited by John Clift and Alexander Heazell

Excerpt

More information

\section{Chapter 1}

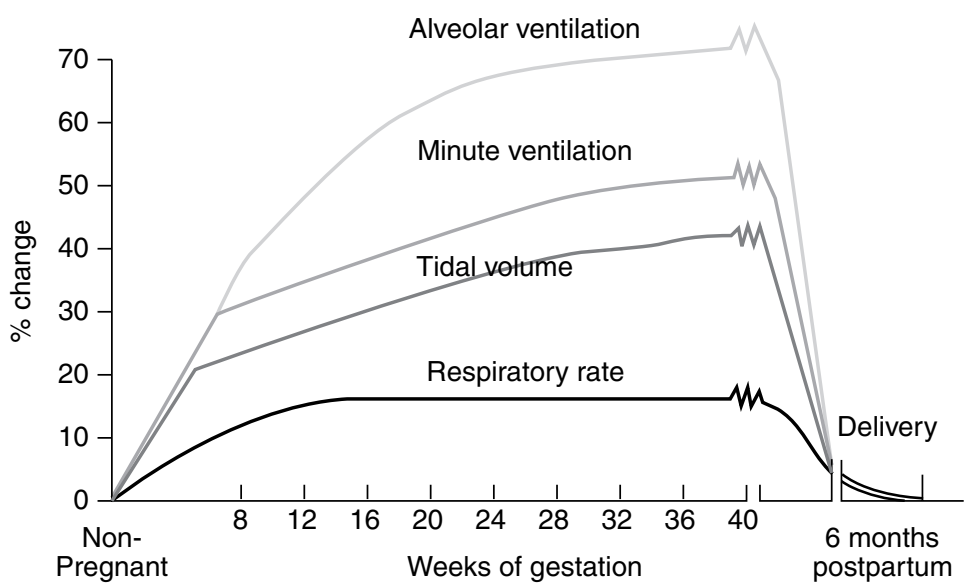

Figure 1.6 Changes in respiratory physiology during pregnancy, showing increased respiratory rate throughout pregnancy, and increasing tidal volume, minute and alveolar ventilation throughout pregnancy. (Reproduced from Buchan and Sharwood-Smith $(1999)^{2}$ with permission from The Surgeon - Journal of the Royal Colleges of Surgeons of Edinburgh and Ireland)

\section{Ventilation}

- $\uparrow$ tidal volume (TV), $\uparrow$ respiratory rate due to raised progesterone levels (Figure 1.6)

- $\downarrow \mathrm{PaCO}_{2}$ to $4.1 \mathrm{kPa}$; this needs maintaining during general anaesthesia, by increasing the minute volume by approximately $40 \%$, to prevent maternal respiratory acidosis

- To compensate $\downarrow \mathrm{HCO}_{3}{ }^{-}(18-21 \mathrm{mmol} / \mathrm{l})$ and base deficit -2 to -3

\section{Hyperventilation}

- During labour, minute ventilation may rise by up to $300 \%$

- Left shift in $\mathrm{O}_{2}$-dissociation curve causes $\uparrow$ maternal affinity for $\mathrm{O}_{2}$ and a $\downarrow$ fetal $\mathrm{O}_{2}$ delivery

- $\downarrow \mathrm{PaCO}_{2}$ may cause vasoconstriction between contractions resulting in a $\downarrow$ placental perfusion

- The above may be helped by epidural analgesia

\section{Intubation in pregnancy}

- Internal diameter of trachea decreased due to capillary engorgement of mucosa, therefore use a smaller endotracheal tube for a similar size non-pregnant woman

- Failed intubations 7-8 times more common in pregnancy, due to capillary engorgement and oedema of upper airway, increased chest diameter, large breasts, 
Cambridge University Press

978-0-521-69670-8 - Obstetrics for Anaesthetists

Edited by John Clift and Alexander Heazell

Excerpt

More information

Maternal physiology and obstetrics

increase in fat deposition, cricoid pressure, left lateral tilt and stress due to time demands

- Nasotracheal intubation and the insertion of nasopharyngeal airways should be avoided due to the increased risk of bleeding

\section{Gastrointestinal system}

- Progesterone-mediated $\downarrow$ LOS pressure and $\uparrow$ intra-abdominal pressure due to gravid uterus cause $\uparrow$ risk of reflux and aspiration of gastric contents

- Gastric emptying normal in pregnancy but delayed in labour and after administration of opiates

- Pregnant patients should be considered to be at risk from aspiration from approximately 16/40 (before if symptoms of reflux)

- Risk of regurgitation reduces to non-pregnant levels within 24-48 hours of delivery, provided there are no symptoms of reflux

\section{Implications of changes in gastrointestinal physiology}

- Patients should be premedicated with an $\mathrm{H}_{2}$-blocking drug the evening before and on the morning of Caesarean section. $30 \mathrm{ml} 0.3 \mathrm{Mol}$ sodium citrate should be given immediately before a rapid sequence induction with cricoid pressure, which is used when administering general anaesthesia.

- Many units have a policy of clear fluids only orally whilst in labour.

\section{Central nervous system}

\section{Regional anaesthesia/analgesia}

- $\uparrow$ lumbar lordosis, makes the interspinous spaces smaller making regional anaesthesia more difficult.

- $\uparrow$ sensitivity of nerves makes onset of block quicker and last longer.

- Distension of epidural veins increases risk of vascular damage/injection.

- 30-50\% $\downarrow$ in doses of local anaesthetics for same block, compared with nonpregnant patients. Amongst proposed reasons are: engorged epidural veins may reduce the size of epidural space and the volume of CSF, swollen veins block the escape of drugs through the vertebral foramina, and cephalad spread due to exaggerated lumbar lordosis.

\section{General anaesthetics}

- MAC value of volatile anaesthetic agents $\downarrow$ by $25-40 \%$

- $\downarrow$ sleep dose of thiopentone by approximately $35 \%$

- The above are due to sedative effects of higher levels of progesterone, $\uparrow$ CNS serotonin activity and activation of endorphin system 


\section{Cambridge University Press}

978-0-521-69670-8 - Obstetrics for Anaesthetists

Edited by John Clift and Alexander Heazell

Excerpt

More information

\section{Chapter 1}

\section{Terms and definitions used in obstetrics}

The specialised nature of obstetrics is associated with terms not used in other fields of medicine, for example, those that are used to describe the number of pregnancies and deliveries. The following list describes terms in common usage in obstetrics:

Gravidity The number of times a woman has been pregnant, irrespective of outcome.

Primigravida A woman who is pregnant for the first time.

Elderly primigravida A woman who is pregnant for the first time and is over 35 years of age.

Parity The number of times a woman has given birth to a fetus of 24 or more weeks gestation, irrespective of outcome/survival/mode of delivery.

Nulliparous A woman who has not given birth to a fetus of 24 or more weeks gestation.

Grandmultiparous A woman who has delivered 4 or more infants of 24 weeks gestation.

\section{Gravida x Para $\mathrm{y}^{+2}$}

$x=$ total number of pregnancies including present pregnancy

$y=$ parity

$z=$ the number of times a woman has been pregnant and not reached 24 weeks gestation e.g. miscarriages, ectopics

$y^{+z}$ excludes the present pregnancy

Example: A woman currently pregnant having had one ectopic, one stillbirth at 27 weeks and one live baby is $\mathrm{G} 4 \mathrm{P} 2^{+1}$.

Antepartum Period between conception and the onset of labour, also known as the prenatal and antenatal period.

Intrapartum Time between the onset of labour and delivery of the placenta; during labour.

Postpartum Six-week period after childbirth during which the mother returns, physiologically, to her non-pregnant state. Also known as the puerperium and postnatal period.

Estimated date of delivery The day on which the baby is 'due', this is 40 weeks gestation. In most units this is now determined by ultrasound scanning in the first trimester; previously it was calculated as 40 weeks from the first day of the woman's last menstrual period.

$\mathrm{X}^{+\mathrm{y}} / 40$ Shorthand method of writing gestation. $X=$ number of weeks, $y=$ number of days. Forty weeks is regarded as term.

Term $37^{+0}-41^{+6}$ weeks' gestation.

Preterm (Premature) $24^{+0}-36^{+6}$ weeks gestation i.e. less than 37 completed weeks' gestation.

Lie Describes the longitudinal axis of the fetus. Longitudinal lie-axis parallel with maternal spine, Transverse lie - axis at $90^{\circ}$ with maternal spine, Oblique lie - in between longitudinal and transverse lie. 
Presentation Part of fetus entering mother's pelvis first. Cephalic presentation head; breech presentation - fetal foot, legs or bottom presents. Other presentations include face, brow, shoulder and compound (hand + presenting part).

Position Describes the position of the presenting part of the fetus with respect to the maternal pelvis. Cephalic presentations are described by the occiput. Occipitoanterior $=$ fetal occiput facing anterior (symphysis), occipito-posterior $=$ fetal occiput facing maternal posterior (sacrum), occipito-transverse $=$ fetal occiput facing maternal side.

Rupture of membranes The membranes of the amniotic sac break releasing amniotic fluid per vagina.

Pre-labour rupture of membranes (PROM) > 37/40 gestation, rupture of membranes prior to onset of labour or contractions.

Preterm pre-labour rupture of membranes (PPROM) $<37 / 40$ gestation, rupture of membranes prior to onset of labour or contractions.

Prolonged rupture of membranes Rupture of membranes more than 24 hours prior to the onset of labour.

Spontaneous rupture of membranes (SROM) > 37/40 gestation, rupture of membranes with or after the onset of labour.

Artificial rupture of membranes (ARM) Intentional rupturing of amniotic membranes with sterile instrument or finger sweep to induce labour. Also known as amniotomy.

External cephalic version (ECV) Procedure to externally rotate a breech presentation to cephalic. The fetal breech is disimpacted and the fetal head manipulated until cephalic. There is a higher success rate after 37 weeks.

Oligohydramnios A reduction in the volume of amniotic fluid surrounding the fetus. This may be caused by rupture of membranes, intrauterine growth restriction and renal/urogenital anomalies in the fetus.

Polyhydramnios Excessive amniotic fluid surrounding the fetus. This may be caused by gastrointestinal or neurological problems in the fetus, or maternal diabetes.

Low-birthweight baby An infant with a birthweight less than $2500 \mathrm{~g}$.

Intrauterine growth restriction (IUGR) An infant that does not attain its predicted growth potential or an infant with a birthweight below the 5th centile for gestational age/ethnicity/parity.

Miscarriage Spontaneous loss of a pregnancy before 24 weeks gestation.

Tocolysis The suppression of uterine contractions.

Neonatal period The first 28 days of life.

\section{Clinical implications}

The following obstetric complications are increased in frequency in: Primigravida

- Pre-eclampsia 


\section{Cambridge University Press}

978-0-521-69670-8 - Obstetrics for Anaesthetists

Edited by John Clift and Alexander Heazell

Excerpt

More information

\section{Chapter 1}

Elderly primigravido and women $>35$

- Miscarriage

- Down's syndrome and chromosomal anomalies

- IUGR

- Premature labour

- Gynaecological disorders e.g. fibroids

- Other diseases e.g. ischaemic heart disease

High parity

- Abnormal fetal presentation

- Obstetric haemorrhage

- Uterine rupture during labour

- Grandmultiparous women have a 6 times increase in mortality rate compared with primigravida women

\section{REFERENCES}

1. G. Chamberlain and M. Morgan, ABC of Antenatal Care, 4th edn (Oxford: Blackwell Publishing, 2002).

2. A.S. Buchan and G.H. Sharwood-Smith The Simpson Handbook of Obstetric Anaesthesia (Edinburgh: Albamedia on behalf of The Royal College of Surgeons of Edinburgh, 1999). www.homepages.ed.ac.uk/asb/.

\section{FURTHER READING}

A.S. Buchan and G.H. Sharwood-Smith, The Simpson Handbook of Obstetric Anaesthesia (Edinburgh: Albamedia on behalf of The Royal College of Surgeons of Edinburgh, 1999). www.homepages.ed.ac.uk/asb/.

D. H. Chestnut, Obstetric Anaesthesia Principles and Practice, 3rd edn (New York: Mosby, 2004).

B. H. Heidemann and J. H. McClure, Changes in maternal physiology during pregnancy. Continuing Education in Anaesthesia, Critical Care and Pain, 3 (2003) 65-8.

I. Power and P. Kam, Maternal and Neonatal Physiology. In I. Power and P. Kam eds., Principles of Physiology for the Anaesthetist (London: Arnold Publishers, 2001). 